Uso de swellable packers en fracturamientos y estimulaciones.

Jorge Ordóñez A., José Villegas S, Carlos Portilla L. 


\title{
Uso de swellable packers en fracturamientos y estimulaciones
}

\section{Use of swellable packers in fractures and stimulations}

\author{
Jorge Ordóñez A. ${ }^{(1)}$, José Villegas S. ${ }^{(1)}$ \& Carlos Portilla L. ${ }^{(1)}$ \\ (1) Facultad de Ciencias de la Ingeniería, Universidad Estatal Península de Santa Elena (UPSE) $\square$ Campus \\ La Libertad, vía principal Santa Elena - La Libertad La Libertad-Ecuador jordonez@upse.edu.ec
}

\begin{abstract}
Resumen
En el presente trabajo se presenta la idea del uso de swellable packers como herramienta adicional, enfocado principalmente en el uso alternativo de cemento. Los swellable packers contienen elastómeros que absorben cierto tipo de fluido, existiendo dos grandes grupos que son: elastómeros con tendencia a fluido con base agua y elastómeros con tendencia a fluido con base aceite. Estos packers fueron creados con el objetivo de usarse como aisladores de zonas, ya que son packers que gracias a sus elastómeros se hinchan al contacto con el fluido al que son a fin, logrando un sello completo en diferentes zonas. Con el pasar de los años se los enfocó en usos diferentes para el que netamente fueron creados, usos como: estimulación de pozos, incluyendo fracturamientos (resistencia a altas presiones), completacion de pozos, trabajos de cementación, etc. Luego del análisis de varios casos y de presentar las ventajas del uso de esta herramienta se propone el empleo de los swellable packers en reemplazo del cemento, esto ayudará a reducir costos de operaciones, tiempo de rig y NPTS ${ }^{1}$.
\end{abstract}

Palabras Clave: Swellable packers, elastómeros de absorción de fluidos, aislamiento de zonas, costo de operaciones.

\begin{abstract}
In this paper the idea of use of swellable packers as alternate tool is introduced. Mainly, focusing of using it as option for the cementation of a well. Swellable packers contains elastomers, which swallow a certain type of fluid. There are two main groups of swellable packers: elastomers which tend to swallow water-based fluid and elastomers which tend to swallow oil-based fluid. Swellable packers were created to solve the problem of isolate different zones. Its elastomer element swallow the fluid and allows to fill up the rubber which creates a good seal between the different zones. Nowdays, swellable packers are used in many different ways such as: well stimulation, includying fracturing (allowing high pressures to be applied to the tool), well completion, cementation jobs, etc. After making the several cases analysis and present the advantages of using this tool, a proposal for the use of swellable packers as a cement's replacemnet had been introduced. This new option would save money in terms of cost operations, rig's time and NPTs.
\end{abstract}

Keywords: Swellable packers, lastomers which absorbs fluid, zone isolation, operational costs.

1 "Non-Productive Time", por sus siglas en ingles. Tiempo no productivo 


\section{Introducción}

Con el creciente aumento de la demanda de energía a nivel mundial, las empresas operadoras se ven en la necesidad de implementar nuevos métodos para el incremento de la producción de hidrocarburos. Sin embargo, con precios bajos por barril de petróleo, se busca aumentar la eficiencia de los recursos que se invierten, es decir producir la mayor cantidad posible con la menor inversión posible.

La industria hidrocarburífera se divide en tres etapas muy bien marcadas: exploración, perforación y producción; siendo la perforación la etapa mas cara del proceso. Dentro de la perforación, el mayor gasto es producido por la utilización de la torre de perforación, es decir el principal objetivo es reducir estas horas de uso del rig que derivará en un ahorro en la perforación y por ende en el costo total del desarrollo de un pozo. Si nos enfocamos netamente en la torre de perforación, nos daremos cuenta que la mayor cantidad de tiempo posible de reducir se encuentra en los procesos tanto de extracción como de introducción de la sarta de perforación, por cualquiera que sea su motivo. La idea de este paper es presentar una alternativa posible para la reducción del tiempo de uso de un rig de perforación al momento de cementar.

Los packers son usados para crear un sello entre zonas. Estos packers pueden ser asentados usando fuerzas hidráulicas, movimientos mecánicos o pulsos eléctricos (Lyons, 2014).

Los REPs ${ }^{2}$ o swellable packers, proporcionan una nueva opción al momento del uso de un packer. Estos packers son comúnmente usados en pozos con multiples zonas de producción a hueco abierto, por su simplicidad y efectividad para estos casos. Sin embargo estos packers tienen otros usos, como por ejemplo: en selección de zonas, completación y fracturamiento.

La tecnología de los swellable packers no necesita de fuerzas hidráulicas ni mecánicas ni ninguna fuerza eléctrica para poder ser asentado (Lyons, 2014). Esta clase de packers utilizas eslastomeros que incrementan su tamaño al contacto con cierto fluido que en la mayoría de los caso es un fluido a base de agua o uno a base de aceite para poder crear el sello entra las zonas. Esta nueva tecnología es mayormente aplicada en pozos donde se busca eliminar la irrupción de agua (Lyons, 2014).

El packer mecánico permanente permanente comúnmente empieza a fallar con el tiempo (AlYami et al., 2008). Existen completaciones que deben ser modificadas para reducir perdidas e incrementar la producción. Esta es una de las principales razones de porque los swellable packers fueron desarrollados (Al-Yami et al., 2008).

En los primeros casos los swellable packers fueron usados para proporcionar aislamiento zonal en pozos sin casing (hueco abierto), usando estos elastómeros que crean un sello entre el hueco y el tubing. Ahora, esta tecnología es aplicada en muchos casos como en operaciones de estimulación, trabajos de cementación y completaciones selectivas (Denney, 2009).

El objetivo de esta investigación is introducir el uso de los swellable packer como una alternativa de cementación para pozos petroleros, teniendo como sustento que estos packers son mucho mas rápidos para su instalación y mucho menos costosos que un trabajo de cementación para la completación de un pozo.

\section{Swellable Packers}

La principal razón para el uso de swellable packers es la reducción del corte de agua. Dado esto, el objetivo será incrementar la producción de hidrocarburos con una menor producción de agua de formación. En otras palabras, producir menos agua mientras se incrementa la producción de hidrocarburos. Con esto en mente, el asilamiento zonal para pozos horizontales se convierte en un reto. Cuando el pozo produce agua a altas tazas, aparecen problemas que pueden conllevar a un incremento en los costos operacionales. Usando swellable packers, puede ser evitado desconectar y extraer el liner con el cemento para aislar las zonas con tazas altas de producción de agua, así mismo estas clases de packers pueden ser corridos tanto en pozos a hueco abierto como en pozos a hueco entubado (Al-Yami et al., 2008).

Los swellable packers utilzan elastómeros como agente sellante, evitando el uso de multiples partes que en el caso de los packers convencionales si contienen. Haciendo uso de esta estrategia se puede disminuir la complejidad de la herramienta y por ende se reduce el riesgo de daño de la misma. Estos packers eliminan el uso de fuerzas hidráulicas, por lo tanto reducen considerablemente el riesgo de roturas de pin producto de altas presiones. De la misma manera evitan el uso de fuerzas mecánicas, que en el caso de una falla generaría un NPT en el trabajo. Por

\footnotetext{
2 "Reactive Element Packers", por sus siglas en ingles. Packers con elemento radiactivo
} 
lo tanto, estos packers trabajan usando el princio de expansión (Evers et al., 2009).

Los swellable packers están formados por tres partes: un nucleo interior que muestra la máxima rata de absorción, un nucleo externo que muestra la minima rata de absorción y una barrera de defusión que tiene un poder de absorción menor que los dos anteriores (Al-Yami et al., 2008).

Para resumir, estas tres partes están contenidas en un caucho el cual absorbe el tipo de fluido especificado que a su vez producirá la expansión de este caucho y creará el sello entre el tubing y el casing o el tubing y el hueco abierto o el hueco abierto y el casing (AlYami et al., 2008).

Sin importar el tipo de elastómero a usarse, la literatura consultada mostró que hay tres tipos de elestómeros. El primer tipo es un elastómero de absorción de aceite y trabaja con el principio de disolución y absorción. Estos elastómeros absorben aceite en el caucho, produciendo una expansión del caucho y creando el sello. El segundo tipo es de un elastómero que absorbe agua y funciona bajo el principio de ósmosis. Este proceso ocurre como resultado de la diferencia de salinidad de los fluidos, empujando el agua (mayor salinidad) a la membrana (menor salinidad), el caucho absorbe el agua, por último este se expande provocando que el sello puede efectuarse. El tercer tipo de elastómero es de un tipo híbrido, este packer puede ser diseñado de diferentes maneras de modo que puede cumplir con las necesidades del trabajo que deba efectuar (Evers et al., 2009).

Actualmente, los swellable packers son usados en varias operaciones, muy lejanas del objetivo principal de su creación. Por ejemplo, son usados como soporte del cemento, como una alternativa de packer en las diferentes completaciones, para propósitos de estimulación y finalmente como una alaternativa de cemento que es el uso que será tratado en este artículo (Evers et al., 2009).

\section{Diseño de un swellable packer}

Como fue mencionado anteriormente, dos grandes grupos de packers existen hoy en día en el mercado: packers de absorción de aceite y packers de absorción de agua (excluyendo la tercera opción de packers híbridos los cuales son menos usados por la complejidad de su uso) (Yakeley et al., 2007). En términos de rapidez de absorción, varias pruebas específicas deben realizarse en las cuales se considerarán importantes elementos tales como: material del caucho, composición química del fluido, temperatura, presión, etc.

Como conocimiento general, para packers de absorción de fluidos basados en agua, se usa agua fresca como fluido de mayor rapidez de absorción producto a su bajo contenido de sal y bajo contenido de contaminantes. Por otro lado, en los packers que absorben fluidos con base de aceite, la mayor rapidez de absorción está en los aceites más livianos en comparación con los aceites más pesados (Yakeley et al., 2007).

Con esto en mente, se mantiene una incognita importante de entender, la cual es la cantidad de tiempo necesaria para obtener la máxima absorción, y se debe tomar en consideración el material del packer y el fluido que absorberá el mismo. Por ejemplo, si un packer es usado en un pozo donde el fluido tiene un bajo potencial de absorción, es posible que el packer no absorba suficiente fluido para ejecutar un buen sello en el hueco (Yakeley et al., 2007).

Antes de seleccionar un packer, hay varias consideraciones que deben ser tomadas en consideración como:

- Material del mandril del empaquetador

- Diámetro externo, $\mathrm{OD}^{3}$

- Longitud del packer

- Temperatura

La figura 1 muestra claramente cómo el diámetro

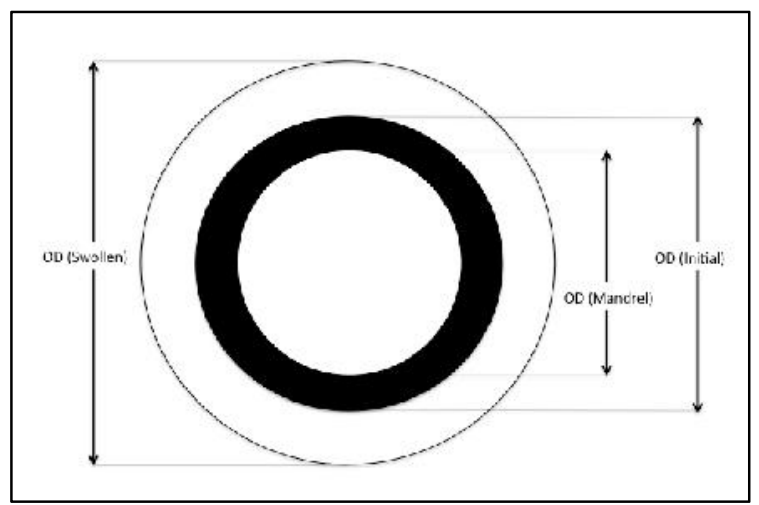

externo es usado para calcular el porcentaje de volumen absorbido, usando las siguientes ecuaciones:

Figura 1 Diagrama de referencia para el volumen de absorción

Fuente: Yakeley et al. (2007)

3 “Outside diameter", por sus siglas en ingles. Diámetro externo 


$$
\begin{gathered}
\% \text { Absorción }=\frac{\left(V_{(\text {absorbido })}-V_{(\text {inicial })}\right)}{V_{(\text {inicial })} * 100} \\
\% A b \operatorname{sorción}=\frac{\left[O D^{2}{ }_{(\text {absorbido })}-O D^{2}{ }_{\text {(inicial })}\right]}{\left[O D^{2}{ }_{\text {inicial })}-O D^{2}{ }_{(\text {mandril })}\right] * 100}
\end{gathered}
$$

\subsection{Prueba de packers con absorción de aceite}

Para considerar el uso de packers de aceite una serie de estudios deben realizarse, ya que de esta manera se puede determinar su utilidad. El principal objetivo de estas pruebas fue proporcionar un escenario preciso para simular los swellable packers. La data de dos pruebas son presentados en la tabla 1:

Tabla 1. Data de las pruebas

Fuente: Data obtenida de (Yakeley et al., 2007)

\begin{tabular}{|l|c|c|}
\hline \# de prueba & 1 & 2 \\
\hline Dimensión & $4.5^{\prime} \times 5.625^{\prime}$ & $4.5^{\prime} \times 5.625^{\prime}$ \\
\hline Casing & $\begin{array}{c}25 \mathrm{a} 26 \\
2-5 / 8^{\prime}, 42.8 \\
\mathrm{lb} / \mathrm{ft}^{4}\end{array}$ & $\begin{array}{c}7-5 / 8^{\prime}, 42.8 \\
\mathrm{lb} / \mathrm{ft}\end{array}$ \\
\hline $\begin{array}{l}\text { Diametro } \\
\text { interno }\end{array}$ & $6.575^{\prime}$ & $6.590^{\prime}$ \\
\hline Llenado con & No.2 Diesel & No.2 Diesel \\
\hline
\end{tabular}

Luego de que fueron realizadas varias pruebas y luego de usar tanto la ecuación 1 como la 2, los resultados del procentaje absorbido fue de $102 \%$ para la prueba 1 y de $103 \%$ para la prueba 2 (Yakeley et al., 2007). Ambas pruebas fueron simulados en el escenario del peor caso usando una situación adversa en la cual un wash-out ${ }^{5}$ significante fue replicado. Las pruebas fueron corridas usando una temperatura de $109{ }^{\circ} \mathrm{F}$ y se le proporcionó tiempo suficiente para absorber fluido hasta sellar el diámetro interno del casing. Luego de que las líneas fueron presurizadas y durante la primera prueba, se observó que a una presión de 4000 psi el fluido hizo un bypass por el packer, mientras que, por otro lado, cuando la presión se estabilizó en 3400 psi, las tazas de perdida fueron reducidas.

Con respecto a la prueba del segundo packer, el comportamiento fue similar. Durante la primera presurización, el packer llegó hasta 3400 psi antes que la primera fuga aparezca. Luego de dos días, el

4 Libras por pie

5 Incremento del diámetro del pozo, causada por la remoción de granos de la formación, durante la perforación o circulación del pozo. packer fue re-presurizado y esta vez la fuga apareció despúes de 3000 psi. Estas pruebas muestran que, en casos ideales, incluso cuando los packers fueron absorbido a procentajes mayores, los packers aún respondieron y fueron capaces de ejecutar su trabajo (Yakeley et al. (2007).

\subsection{Instalación de un swellable packer}

Luego de que la decisión del uso de swellable packers se haya tomado, la compañía de servicio debe preparar la herramienta que fue solicitada para el trabajo. Generalmente, la compañía hace una simulación para poder predecir el comportamiento de la fromación posterior a la decisión de qué tipo de swellable packer debería ser utilizado. Un típico procedimiento para correr un swellable packer procede como se detalla a continuación:

1. Planeamiento y preparacion pre-trabajo, de modo que la mejor herramienta sea seleccionada para el trabajo.

2. Recolectar toda la información necesaria para el buen diseño de la herramienta. Esta data debe incluir aspectos importantes como:

- Tamaño del pozo

- Trayectoria

- Configuración de la completación

- Tamaño de sarta, grado, peso, longitud y tipo de rosca

- Fluidos de reservorio

- Presión de fondo de pozo

- Temperatura circulante de fondo de pozo

- Temperatura estatática de fondo de pozo

3. Seleccionar el equipo apropiado para las operaciones esperadas de completación.

4. Instalar los elementos flotadores.

5. Correr el tubing con los swellable packers adheridos.

6. Circular el fluido correcto para comenzar el proceso de absorción.

7. Esperar hasta que el swellable packer absorba la cantidad de fluido suficiente para hacer sello (este tiempo es predecido usando simulaciones e utilizando un margen de error suficiente para logra un buen asentamiento). 
8. Empezar la transición a superficie del equipo de completación.

Al final de este proceso, el pozo debe ser presurizado para asegurarse que el swellable packer hizo un buen sello en el pozo (Rogers et al., 2008).

\section{Swellable packer como método de completación}

\subsection{Completaciones convencionales}

Para poder completar un pozo, hay tres tipos de métodos estándares. El primer método es el de hueco abierto, este método es usado cuando el pozo esta constituido de formaciones duras (es decir pocas probabilidades de colapso en el pozo); es una completación económicamente efectiva, simple y rápida. Por estas razones es el método más efectivo en términos de producción, ya que no hay restricciones para el fluido en alcanzar el pozo (Rogers et al., 2008).

Un segundo método es usando un liner perforado. Este método es básicamente el mismo método que el de hueco abierto, pero con la diferencia que usa un liner para prevenir la producción de partículas (cuando la formación no es lo suficientemente dura). Típicamente, el liner es colgado desde el fondo del último tubo de la completación (Rogers et al., 2008).

El tercer método convencional y probablemente el más usado a nivel mundial es cementando el casing de producción (Rogers et al., 2008). Este método es más complicado que los dos métodos discutidos anteriormente ya que requiere adeherir cemento al pozo. Usando este método, los riesgos pueden aumentar en términos de una buena lechada de cemento. Así mismo, cálculos exactos deben ser realizados de la cantidad suficiente de cemento a ingresar a las líneas previo a la cementación. Ya que, más bombas y mayor presión debe ser manejadas para realizar un buen trabajo de cementación, el riesgo también se aumenta producto de estos trabajos (Rogers et al., 2008). El casing es idealmente colocado por el resto de la vida del pozo, ya que el reemplazo del mismo es no solo altamente costoso sino que también muy peligroso. Se utiliza un tubing para proteger el casing mientras se desgasta el tubing (el cual puede ser reemplazado sin inconveniente) (Rogers et al., 2008).

La figura 2 muestra las tres diferentes completaciones, donde se puede observar tanto la completación a hueco abierto, liner perforado y cementada.
Figura 2. (a) Completación a hueco abierto, (b)

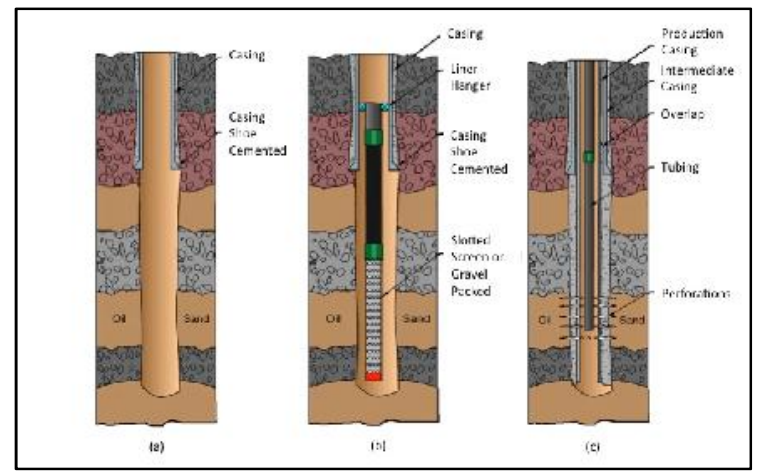

Completación con liner perforado, (c) Completación cementada

Fuente: (Rogers et al., 2008).

Estos tres métodos han sido usados por muchos años generando buenos resultados, pero la tecnología está siempre en la búsqueda de nuevos métodos, los cuales son amigables con el ambiente y más baratos que a su vez representan buenas noticias para el sector petrolero.

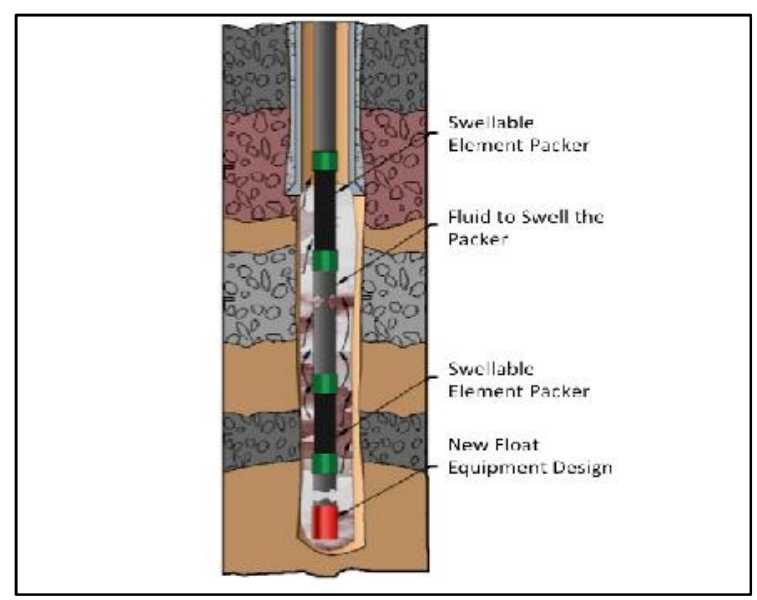

Figura 3. Completación con swellable packer.

Fuente: (Rogers et al., 2008).

\section{Casos de estudio}

\subsection{Caso 1: Campo North Cormorant, UK. North Sea. 2003}

Reto: Aislar la arena productora de agua y producir de la arena que contenga petróleo. 
Este campo se caracteriza por ser altamente compartamentalizado. Previos pozos fueron completados usando liners de 2-7/8", usando cemento para ascentarlos y perforando la zona de pago. Cementar en un agujero pequeño con un pequeño liner hace el trabajo mas difícil e incrementa el riesgo de falla. Por esta razón Shell E\&P decidió usar swellable packers para correr una combinación con liner perforado. La inclusión de esta tecnología redujo el costo de operación dado la ausencia de trabajo de cementación, la reducción de tiempo de operación y la eliminación de operación de perforación.

Para este trabajo se usaron 12 swellable packers, los cuales variaron desde 10 a 16 pies de largo con un diámetro externo de 4.2" (Staff, 2009).

\subsection{Caso 2: Onshore, UK}

Reto: Correr un liner perforado de 4-1/2" y asentarlo en un pozo de hueco abierto horizonal de 6".

La empresa de servicios TAM Internacional, ofreció correr un $\mathrm{CAP}^{6}$ por encima del liner. El objetivo de estas completación era usar un swellable packer en vez de cemento. Los CAPs fueron adheridos al liner, donde estos formaban un sello hidráulico entre las paredes del pozo y el liner. Este sello hidráulico trabajó como cemento y el liner pudo ser liberado para continuar las operaciones en este pozo (TAM, 2014).

\subsection{Caso 3: Onshore, Norte de Holanda}

Reto: Correr un swellable packer de 5.750' para aislar una zona productora de casi el $100 \%$ de agua en un casing de 7'.

Este trabajo fue ejecutado usando coiled tubing en un pozo dual lateral de gas. La tecnología del swellable packer, fue escogida para proveer de asilamiento zonal y a su vez evitar la usurpación de agua. Se usaron elastómeros con base en agua, permitiendo la absorción de agua fresca hasta que el sello se haya creado. En este trabajo se instaló una completación con liner y se usó dos swellable packers.

El equipo usó una semana de tiempo de absorción para que los packers obtengan su expansión máxima, aunque el tiempo simulado era menos de dos días, debido a la preocupación de que el packer no iba a mantener su expansión, producto de la presencia de aceite. Por esta razón la compañía decidió usar un swellable packers de mayor diámetro externo para

6 “Casing Annulus Packer", por sus siglas en ingles. Packer de anular y casing

7 "Inside diameter", por sus siglas en ingles. Diámetro interno con esto asegurar un buen sello y un buen asilamiento.

Luego de correr los swellable packers con elastómeros a base de agua, que absorbían agua con una salinidad máxima de 0.50 , se obtuvieron buenos resultados. Los resultados de este caso de estudio se presentan a continuación en la tabla 2 :

\section{Tabla 2. Resultados de aplicación de swellable packers}

Fuente: (Brooks, 2011)

\begin{tabular}{|l|c|c|c|}
\hline Restricción & Mínimo ID $^{7}$ & 6.184 & $\mathrm{In}^{8}$. \\
\hline Sello & Mínimo ID & 6.184 & $\mathrm{In}$. \\
\hline Sello & Mínimo ID & 6.247 & $\mathrm{In}$. \\
\hline Elastómero pre-absorción & OD & 5.750 & $\mathrm{In}$. \\
\hline Elastómero pre-absorción & ID & 4.5 & $\mathrm{In}$. \\
\hline Presión requerida & Nominal & 1500 & $\mathrm{Psi}^{9}$. \\
\hline Packer luego de absorción & Máximo OD & 7.231 & $\mathrm{In}$. \\
\hline $\begin{array}{l}\text { Sello equivalente } \\
\text { descentralizado }\end{array}$ & Mínimo ID & 4.5 & $\mathrm{In}$. \\
\hline $\begin{array}{l}\text { Sello equivalente } \\
\text { descentralizado }\end{array}$ & Máximo ID & 4.5 & $\mathrm{In}$. \\
\hline
\end{tabular}

Ambos packers, tanto superior como inferior fueron corridos con éxito y ellos aislaron la zona de agua. Los packers, según el software de simulación debiando completar su absorción luego de 24 horas en contacto con el fluido. Sin embargo, el equipo decidió exponerlos al contacto con el fluido por aproximandamente 7 días, para con esto asegurar que se expandieron correctamente y evitar errores en los cálculos que provoquen fallas en el sello. Los packers se presurizaron y sostuvieron una presión de 580 psi de presión diferencial (Brooks, 2011).

\subsection{Caso 4: Placa continental de Noruega}

Reto: Correr un tubing de 4-1/2" y asentarlo en un liner de 7".

La compañía de servicio Halliburton, ofreció correr un swellable packer por encima del tubing y otro por debajo. Este swellable packer funcionó como sello entre ambos el hueco abierto-tubing y el liner-tubing. Esto produjo que el colgador de liner hiiera un torque el cual permitió que el tubing sea removido (Halliburton, 2015b).

\section{Conclusiones y recomendaciones}

Los swellable packers son herramientas muy confiables en términos de seguridad en cuanto a la

8 Abreviación del ingles "inches". Pulgadas

9 Abreviación del ingles "Pounds per Square Inches". Libras por pulgadas cudradas 
presión. Estos han demostrado que son muy útiles en el uso de trabajos como fracking, los cuales dentro de la industria son los que causan retos más grandes a los ingenieros de la industria. Si estos packers logran reemplazar al cemento, se hablaría de ahorros bastante significativos en el costo de una perforación de un pozo petrolero.

Por lo cual se debería considerar un estudio más profundo sobre el tema y sobre la posible utilización de esta herramienta como sustituto del cemento. Además, se debería simular varios casos en los cuales se ponga a prueba los swellable packers a situaciones extremas y corroborar el rango de uso de esta herramienta.

\section{Referencias}

[1] AL-YAMI, A., NASR-EL-DIN, H. \& ALHUMAIDI, A. 2008. Investigation of Water-Swelling Elastomers: Advantages, Limitations, and Recommendations. 2008

[2] BROOKS, R. 2011. Merging Coiled Tubing and Swellable Packer Technologies. 2011 2011. Texas.

[3] DENNEY, D. 2009. Designing SwellableElastomer Packers in Fracturing Operations. Journal of Petroleum Technology, 61, 5861.

[4] EVERS, R., YOUNG, D., VARGUS, G. \& SOLHAUG, K. 2009. Designing swellableelastomer packers in fracturing operations. JPT, Journal of Petroleum Technology, 61, 58-61.

[5] HALLIBURTON. 2015a. Swellable Packers [Online]. Available: http://www.halliburton.com/enUS/ps/completions/wellcompletions/swelltechnology/default.page?node$\mathrm{id}=$ hfqel9w7.

[6] HALLIBURTON. 2015b. Swellpacker ${ }^{\circledR}$ system is used as an anchor to provide torque for liner hanger running tool [Online]. Halliburton. Available: http://www.halliburton.com/public/cps/con tents/Case_Histories/web/H011860-

Emergency-Release.pdf.

[7] LYONS, W. 2014. Working Guide to Drilling Equipment and Operations. Elsevier Science.

[8] ROGERS, H., ALLISON, D. \& WEBB, E. New Equipment Designs Enable Swellable
Technology in Cementless Completions. 20082008 Texas.

[9] SCHULUMBERGER. 2015. Swellable Packers [Online]. Available: http://www.slb.com/services/completions/p ackers/swellable.aspx.

[10] STAFF, J. P. T. 2009. Versatile Swellable Packer Tool Provides Reliable and Economical Zonal Isolation. Journal of Petroleum Technology, 61, 26-33.

[11] SWELL-X. 2015. X-Packer [Online]. Available: $\quad$ http://www.swell-X.com/Xpacker.php.

[12] TAM. 2014. Swellable Packers [Online]. Available: http://www.tamintl.com/products/swellable -packers/overview-7.html.

[13] TENDEKA. 2015. Swellable Packer [Online]. Available: http://www.tendeka.com/products/swellfixpacker/.

[14] WEATHERFORD. 2015. Annular Swellable Packers [Online]. Available: http://www.weatherford.com/productsservices/well-construction/zonalisolation/swellable-technologies/annularswellable-packers.

[15] YAKELEY, S., FOSTER, T. \& LAFLIN, W. 2007. Swellable Packers for Well Fracturing and Stimulation. November 2007 2007. 1-7. 